\title{
DOUBLY SUPERSYMMETRIC NULL STRINGS AND STRING TENSION GENERATION
}

\author{
Igor A. Bandos \\ Kharkov Institute of Physics and Technology \\ Kharkov, 310108, the Ukraine

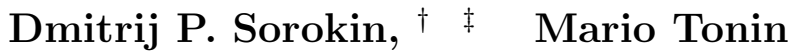 \\ Dipartimento di Fisica "G. Galilei" \\ Università degli Studi di Padova, Italy \\ Dmitrij V. Volkov \\ Kharkov Institute of Physics and Technology \\ Kharkov, 310108, the Ukraine
}

DFPD $/ 93 / \mathrm{TH} / 48$

July 1993

\begin{abstract}
We propose a twistor-like formulation of $\mathrm{N}=1, \mathrm{D}=3,4,6$ and 10 null superstrings. The model possesses $\mathrm{N}=1$ target space supersymmetry and $n=\mathrm{D}-2$ local worldsheet supersymmetry, the latter replaces the $\kappa$-symmetry of the conventional approach to the strings. Adding a Wess-Zumino term to a null superstring action we observe a string tension generation mechanism [1, 2]: the induced worldsheet metric becomes nondegenerate and the resulting model turns out to be classically equivalent to the heterotic string.
\end{abstract}

\footnotetext{
†Supported in part by the European Community Research Program "Gauge Theories, Applied Supersymmetry and Quantum Gravity" under contract CEE-SCI-CT92-0789

${ }^{\ddagger}$ Permanent address: Kharkov Institute of Physics and Technology, Kharkov, 310108, the Ukraine e-mail address: kfti\%kfti.kharkov.ua@relay.ussr.eu.net
} 
Null strings [3] have attracted certain attention from various points of view [4, 5, 6,

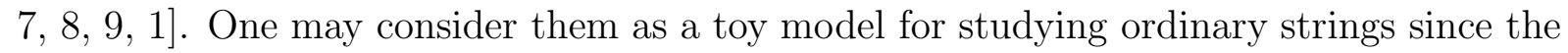
former, being characterized by the zero tension and degenerate worldsheet metric, take an intermediate position between the particles and the strings. Actually, the null strings are closer to the particles than to the strings because they just describe a collection of particles moving on a null surface [4, 9]. Moreover the null strings do not need any critical dimension of space-time for living in [6, 7] and the null superstrings [4, 8, 9], in contrast to the ordinary superstrings, do not require a Wess-Zumino term for getting the fermionic $\kappa$-symmetry, which is necessary to get rid of redundant fermionic degrees of freedom (see 111 for a review on strings).

From a physical point of view the most interesting fact is an assumption that the null string may be regarded as a high energy limit of the string [5, 9], thus providing a way for describing strings beyond the Planck's scale.

Here, performing a program of "twistorizing everything" [12]- [20], [2] we propose a twistor-like formulation of $\mathrm{N}=1, \mathrm{D}=3,4,6$ and 10 null superstrings. The model possesses $\mathrm{N}=1$ target space supersymmetry and $n=\mathrm{D}-2$ local worldsheet supersymmetry, the latter replaces the $\kappa$-symmetry of other approaches 1 .

We shall see that for the doubly supersymmetric null string action to be super reparametrization invariant one needs only a superfield containing a bosonic "einbein". This einbein characterizes the geometry of the bosonic part of the null super worldsheet [1, 7, 9]. So the worldsheet gravitino and $\mathrm{O}(\mathrm{n})$ gauge field drop out of the null string action.

The geometry of the null super worldsheet [9, 10] resembles that of a spinning particle worldline [22] from the one-hand side and the heterotic worldsheet geometry [15, 2] from the other. The action of the model under consideration turns out to be closely related to a "geometro-dynamical" part (in terminology of Galperin and Sokatchev [18]) of a heterotic string twistor-like action [15, 2]. The former arises as a singular solution of twistor-like heterotic string constraints which corresponds to a degenerate worldsheet metric and requires the string tension to be zero?.

On the contrary, adding a Wess-Zumino term generating the string tension to the null superstring action is similar to a tension generation mechanism proposed in [1, 2]. Note that earlier an alternative way of generating the string tension was considered in [5].

Our starting point is a twistor-like action for a bosonic null string written in a first-

\footnotetext{
${ }^{1}$ Earlier various doubly supersymmetric models were considered in [21] irrelevant to the $\kappa$-symmetry problem.

${ }^{2}$ For $\mathrm{N}=2, \mathrm{D}=3$ Green-Schwarz superstring a similar singular solution was considered in [18]. But since the authors imposed too rigorous gauge fixing they associated the solution with a superparticle and not a null superstring.
} 
order formalism:

$$
S=\int d^{2} \xi p_{m}\left(V^{\mu} \partial_{\mu} x^{m}-\bar{\lambda} \gamma^{m} \lambda\right),
$$

where $\xi^{\mu}(\mu=0,1)$ parametrize a null surface whose geometry is determined by an "einbein" $V^{\mu}$ (see [9] for the details), $x^{m}$ and $p_{m}$ are, respectively, the null string coordinate and the momentum density in $\mathrm{D}=3,4,6$ or 10 dimensional target space-time $(\mathrm{m}=0,1, \ldots, \mathrm{D}-$ 1 ), and $\lambda^{\alpha}$ is a Majorana or Majorana-Weyl commuting spinor (twistor component) depending on the dimension of the target space.

Action (11) is a generalization of the twistor-like particle action [12, 17], and can be derived from other formulations of the null string [4, 7, 9].

To show that eq. (国) indeed describes a bosonic null string we solve the equations of motion:

$$
\begin{gathered}
\partial_{\mu}\left(V^{\mu} p_{m}\right)=0, \\
V^{\mu} \partial_{\mu} x^{m}-\bar{\lambda} \gamma^{m} \lambda=0, \\
p_{m}\left(\bar{\lambda} \gamma^{m}\right)_{\alpha}=0 \rightarrow p_{m}=a(\xi) \bar{\lambda} \gamma_{m} \lambda, \\
p_{m} \partial_{\mu} x^{m}=0,
\end{gathered}
$$

where (3) represents the Cartan relation for the lightlike vector in $\mathrm{D}=3,4,6$ and 10 . From eqs. (2) -(团) it follows that

$$
V^{\mu} \partial_{\mu} x^{m} \partial_{\nu} x_{m}=0 .
$$

This means that the induced worldsheet metric $G_{\mu \nu}=\partial_{\mu} x^{m} \partial_{\nu} x_{m}$ is degenerate, which is a peculiar property of the null string.

If we introduce intrinsic degenerate metric $g_{\mu \nu}$ on the null string

$$
V^{\mu} g_{\mu \nu}=0
$$

eqs. (5) and (6) mean that $G_{\mu \nu}$ and $g_{\mu \nu}$ coincide up to a scalar factor. The degeneracy of the metric implies that there exists a vector $\Omega_{\mu}$ such that

$$
V^{\mu} \Omega_{\mu}=0 \quad \text { and } \quad g_{\mu \nu}=\Omega_{\mu} \Omega_{\nu} .
$$

One may also assume that on the worldsheet there live vectors $V_{\mu}$ and $\Omega^{\mu}$ inverse to $V^{\mu}$ and $\Omega_{\mu}$, respectively:

$$
V^{\mu} V_{\mu}=1=\Omega_{\mu} \Omega^{\mu}
$$

We use all these vectors to construct a tension generating term in the action of null superstring. Note that a priori we do not require these vectors to form a non-degenerate intrinsic metric on the worldsheet. Now let us show how a tension generating mechanism works for the simple case of the bosonic null string. Following Refs. [1], 2] we introduce 
an "electromagnetic" field $A_{\mu}$ on a null surface and put it into the action (1) as part of the following term

$$
S=\int d^{2} \xi\left[p_{m}\left(V^{\mu} \partial_{\mu} x^{m}-\bar{\lambda} \gamma^{m} \lambda\right)+\phi \epsilon^{\mu \nu}\left(\partial_{\mu} A_{\nu}-V_{\mu} \Omega_{\nu}\left(V^{\lambda} \partial_{\lambda} x^{m}\right)\left(\Omega^{\rho} \partial_{\rho} x_{m}\right)\right)\right]
$$

where $\phi(\xi)$ is a Lagrange multiplier and $\epsilon^{12}=-\epsilon^{21}=1$. Varying (9) with respect to $\phi$ we just find that the strength of $A_{\mu}$ is:

$$
F \equiv \epsilon^{\mu \nu} \partial_{\mu} A_{\nu}=\epsilon^{\mu \nu} V_{\mu} \Omega_{\nu}\left(V^{\lambda} \partial_{\lambda} x^{m}\right)\left(\Omega^{\rho} \partial_{\rho} x_{m}\right)
$$

Varying with respect to $A_{\mu}$ gives

$$
\partial_{\mu} \phi=0 \rightarrow \phi=T
$$

where $T$ is a constant identified with the string tension [1, 2]. Substituting (11) into the action (9) and skipping the total derivative term we get:

$$
S=\int d^{2} \xi\left[p_{m}\left(V^{\mu} \partial_{\mu} x^{m}-\bar{\lambda} \gamma^{m} \lambda\right)-T \epsilon^{\mu \nu} V_{\mu} \Omega_{\nu}\left(V^{\lambda} \partial_{\lambda} x^{m}\right)\left(\Omega^{\rho} \partial_{\rho} x_{m}\right)\right]
$$

Observe that eq. (9) acquires additional symmetry found in [15:

$$
\delta p_{m}=\phi \epsilon^{\mu \nu} V_{\mu} \Omega_{\nu} A(\xi)\left(V^{\mu} \partial_{\mu} x^{m}+\bar{\lambda} \gamma^{m} \lambda\right) \quad \delta \Omega^{\rho}=-A(\xi) V^{\rho}, \quad \delta V^{\mu}=0
$$

This symmetry allows one to eliminate $p_{m}$ on the mass shell (2), (3). With this in mined, from the variation of (12) with respect to $V^{\mu}$ (and taking into account (8)) one gets the second Virasoro condition (the first one follows from (2)):

$$
\Omega^{\mu} \partial_{\mu} x_{m} \Omega^{\rho} \partial_{\rho} x^{m}=0
$$

As a result the induced metric $G_{\mu \nu}=\partial_{\mu} x^{m} \partial_{\nu} x_{m}$ is not degenerate anymore, and the null string is transformed into an ordinary bosonic string, with $V_{\mu}=e_{\mu}^{+}, \Omega_{\mu}=e_{\mu}^{-}$being identified with the nondegenerate worldsheet zweibeins. Upon dropping the $p_{m}$ term out of (12) one restores the conventional bosonic string action.

Let us proceed now with null superstrings. To get an action for a null superstring we extend the null worldsheet geometry to a geometry on a null super worldsheet parametrized by $\xi^{\mu}$ and by $\mathrm{n}=\mathrm{D}-2$ odd variables $\eta^{a}(a=1, \ldots, n)$, and consider $p_{m}, x^{m}, V^{\mu}$ and $\lambda^{\alpha}$ as components of worldsheet superfields to be introduced below. Note that on the null $\mathrm{d}=2$ surface the spinors are actually scalars, so the letters $a, b, c, .$. from the beginning of the Latin alphabet correspond to the local $O(n)$ group indices [9, 10].

In contrast to Ref. [10, where a number of null $d=2$ superspaces were studied in detail, our choice of constraints on the super worldsheet vielbeins is unique for all $n$-extended supersymmetries and reads as follows

$$
\left\{\nabla_{a}, \nabla_{b}\right\}-\left(E^{-1}\right)_{c}^{d}\left(\nabla_{d} E_{\{a}^{c}\right) \nabla_{b\}}=\delta_{a b} \Delta
$$


where $\nabla_{a}=E_{a}^{b} \partial_{b}+E_{a}^{\mu} \partial_{\mu}, \Delta=E^{\mu} \partial_{\mu}+E^{b} \partial_{b}$, are the supercovariant spinor and vector derivative, respectively, and $E_{a}^{b}, E^{b}, E_{a}^{\mu}, E^{\mu}$ are the components of the super worldsheet vielbeins; $\{\ldots\}$ denotes symmetrization. The second term in the left-hand side of (15) corresponds to an $O(n)$ connection in the tangent space to the null super worldsheet. Eq. (15) allows one to express $E^{a}$ and $E^{\mu}$ in terms of $E_{a}^{b}$ and $E_{a}^{\mu}$.

Redefining $E_{a}^{\mu}$ as

$$
E_{a}^{\mu} \equiv i E_{a}^{b} V_{b}^{\mu}
$$

and using superdiffeomorphisms and local $O(n)$ transformations we can restrict the symmetry transformations on the null super worldsheet to supergravity transformations by choosing a gauge analogous to that used in the case of spinning particles [22]:

$$
E_{a}^{b}=\delta_{a}^{b} E(\xi, \eta)
$$

where $E(\xi, \eta)$ is a superfield containing a gravitino field $\chi_{i}(\xi)$ and $O(n)$ gauge field $A_{[i j]}(\xi)$ as components. (In the null superspace $\chi_{i}$ does not carry vector indices [9, 10].

With eqs. (16) and (17) the constraints (15) are reduced to

$$
D_{a} V_{b}^{\mu}+D_{b} V_{a}^{\mu}=\frac{2}{n} \delta_{a b} V_{c}^{\mu}
$$

where

$$
D_{a}=\partial_{a}+i V_{a}^{\mu} \partial_{\mu}, \quad\left\{D_{a}, D_{b}\right\}=\frac{2 i}{n} \delta_{a b}\left(D_{c} V_{c}^{\mu}\right) \partial_{\mu} .
$$

The local supersymmetry transformations look as follows

$$
\begin{array}{r}
\delta \eta_{a}=-\frac{i}{2} D_{a} \Lambda(\xi, \eta), \quad \delta \xi^{\mu}=\Lambda^{\mu}(\xi, \eta)-\frac{1}{2} V_{a}^{\mu} D_{a} \Lambda \\
\delta V_{a}^{\mu}=-i D_{a} \Lambda^{\mu}+\frac{i}{2}\left(D_{b} \Lambda\right)\left(D_{a} V_{b}^{\mu}\right), \quad \delta D_{a}=\frac{i}{2}\left(D_{a} D_{b} \Lambda\right) D_{b}
\end{array}
$$

where $\Lambda(\xi, \eta)$ and $\Lambda^{\mu}(\xi, \eta)$ are superfield parameters.

In a Wess-Zumino gauge

$$
\left.D_{a}\right|_{\eta=0}=\partial_{a}
$$

with the use of local symmetry parameters

$$
\left.D_{\left[a_{1}\right.} D_{\left.b_{1}\right]} \Lambda^{\mu}\right|_{\eta=0}, \ldots,\left.D_{\left[a_{1}\right.} D_{a_{2}} \ldots D_{\left.a_{n-1}\right]} \Lambda^{\mu}\right|_{\eta=0}
$$

one may put all components of $V_{a}^{\mu}$ to zero except

$$
\left.D_{a} V_{a}^{\mu}\right|_{\eta=0}=V^{\mu}
$$

where $V^{\mu}$ is the null worldsheet einbein (eq. (1)). Note that $V_{a}^{\mu}$ is a generalization of an $E_{\alpha}^{+}$superfield used in [2] for defining heterotic geometry. 
Now we are ready to write down a doubly supersymmetric generalization of the null string action (11). It looks very much like a twistor-like superparticle action [12, 17] and/or heterotic string action [15, 2] without a Wess-Zumino part:

$$
S_{N}=\int d^{2} \xi d^{n} \eta \mathrm{P}_{a m}\left(D_{a} X^{m}-i D_{a} \bar{\Theta} \gamma^{m} \Theta\right)
$$

In (23) a bosonic superfield $X^{m}\left(\left.X^{m}\right|_{\eta=0}=x^{m}\right)$ and a fermionic superfield $\Theta^{\alpha}\left(\left.\Theta^{\alpha}\right|_{\eta=0}=\right.$ $\left.\theta^{\alpha},\left.D_{a} \Theta^{\alpha}\right|_{\eta=0}=\lambda_{a}^{\alpha}\right)$ transform as scalars under the local supersymmetry transformations (20), and the Lagrange multiplier superfield $\mathrm{P}_{a m}$ transforms in such a way that the action (23) is invariant under the local supersymmetry transformations.

Observe that the superfield (17) dropped out of the action, thus the latter does not contain the gravitino and the $O(n)$ gauge field. It is amusing that space-time supersymmetry naturally arises as a consequence of the worldsheet supersymmetrization of eq. (1). Thus, once again worldsheet geometry proves itself to be more fundamental than the target space one. So eq. (23) is also invariant under the global supersymmetry transformations in the target space

$$
\delta \Theta^{\alpha}=\varepsilon^{\alpha}, \quad \delta X^{m}=\bar{\Theta} \gamma^{m} \varepsilon,
$$

and local transformations of the Lagrange multiplier

$$
\delta \mathrm{P}_{a m}=\left(D_{b}+i \partial_{\mu} V_{b}^{\mu}\right) \bar{\Sigma}_{a b c} \gamma^{m} D_{c} \Theta
$$

where $\Sigma_{a b c}^{\alpha}$ is totally symmetric and traceless with respect to the $O(n)$ indices.

Following the reasoning course performed in detail for superparticles [17] and heterotic strings [15, 2] one may derive that all higher components of $X^{m}$ and $\Theta^{\alpha}$ are expressed in terms of leading components, and the only component of $\mathrm{P}_{a m}$ which survives gauge fixing (25) and is not equal to zero due to equations of motion

$$
\begin{gathered}
\left(D_{a}+i \partial_{\mu} V_{a}^{\mu}\right) \mathrm{P}_{a m}=0 \\
\left(\mathrm{P}_{a m} D_{a} \bar{\Theta} \gamma^{m}\right)_{\alpha}=0
\end{gathered}
$$

is the null string momentum density

$$
p_{m}=\left.\varepsilon_{a a_{1} \ldots a_{n-1}}\left(D_{a_{1}}+i \partial_{\mu} V_{a_{1}}^{\mu}\right) \ldots\left(D_{a_{n-1}}+i \partial_{\mu} V_{a_{n-1}}^{\mu}\right) \mathrm{P}_{\mathrm{am}}\right|_{\eta=0} .
$$

Thus, in the Wess-Zumino gauge (21), (22) the action (23) is reduced to the following component form:

$$
S=\int d^{2} \xi p_{m}\left(V^{\mu}\left(\partial_{\mu} x^{m}-i \partial_{\mu} \bar{\theta} \gamma^{m} \theta\right)-\frac{1}{n} \bar{\lambda}_{a} \gamma^{m} \lambda_{a}\right)
$$

\footnotetext{
${ }^{3}$ For simplicity we consider flat geometry in the target superspace, though the generalization to the case of a supergravity background is straightforward
} 
where $\lambda_{a}^{\alpha}$ are subject to the constraint [17]

$$
\bar{\lambda}_{a} \gamma^{m} \lambda_{b}=\frac{1}{n} \delta_{a b} \bar{\lambda}_{c} \gamma^{m} \lambda_{c}
$$

Then eliminating the $\lambda$-term by use of eq. (3), integrating over $p_{m}$ and rescaling $V^{\mu}$ we get a form of the null superstring which one may find, for example, in Refs. [9]:

$$
S=\int d^{2} \xi V^{\mu} V^{\nu}\left(\partial_{\mu} x^{m}-i \partial_{\mu} \bar{\theta} \gamma^{m} \theta\right)\left(\partial_{\nu} x^{m}-i \partial_{\nu} \bar{\theta} \gamma^{m} \theta\right)
$$

The action (30) is invariant under $\kappa$-symmetry transformations [5, 9]:

$$
\begin{gathered}
\delta_{\kappa} \theta^{\alpha}=i V^{\mu}\left(\partial_{\mu} x^{m}-i \partial_{\mu} \bar{\theta} \gamma^{m} \theta\right) \gamma_{m \beta}^{\alpha} \kappa^{\beta}, \quad \delta_{\kappa} x^{m}=i \bar{\theta} \gamma^{m} \delta_{\kappa} \theta \\
\delta_{\kappa} V^{\mu}=2 V^{\mu} V^{\nu} \partial_{\nu} \bar{\theta} \kappa .
\end{gathered}
$$

Using the twistor condition $V^{\mu}\left(\partial_{\mu} x^{m}-i \partial_{\mu} \bar{\theta} \gamma^{m} \theta\right)=\frac{1}{n} \bar{\lambda}_{a} \gamma^{m} \lambda_{a}$ we may replace the parameter $\kappa^{\beta}$ by a parameter $\left.D_{a} \Lambda\right|_{\eta=0} \equiv \epsilon_{a}=\bar{\lambda}_{a} \kappa$ so that, in particular,

$$
\delta \theta^{\alpha}=\epsilon_{a} \lambda_{a}^{\alpha} ; \quad \delta x^{m}=i\left(\bar{\theta} \gamma^{m} \lambda_{a}\right) \epsilon_{a}
$$

In eqs. (32) one may recognize the on-shell $n=D-2$ local supersymmetry transformations (20) of the $\theta^{\alpha}$ and $x^{m}$ components, which allow one to eliminate half of the fermionic degrees of freedom of the null superstring. Thus, $n=D-2$ extended local supersymmetry of the null super worldsheet takes upon itself the role of the $\kappa$-symmetry, and is in fact more fundamental than the latter. This opens the possibility of solving the problems connected with the infinite irreducibility of the $\kappa$-symmetry constraints.

We have already mentioned above that the null superstring action (23) may be obtained from the twistor-like heterotic string action [15, 2] by taking an appropriate zero tension limit which results in vanishing of the heterotic string Wess-Zumino term.

Let us now perform an inverse procedure by adding to eq. (23) a Wess-Zumino term generalizing that of the bosonic model (9) in such a way that the heterotic string action is restored. This is achieved by the string tension generating mechanism of Refs. [1, 2].

The Wess-Zumino part of the action is written as follows:

$$
S_{W Z}=\int d^{2} \xi d^{n} \eta \mathrm{P}^{\mathcal{M N}}\left(\partial_{\mathcal{M}} A_{\mathcal{N}}+B_{\mathcal{M N}}-F_{\mathcal{M N}}\right)
$$

where $\mathcal{M}, \mathcal{N}$ stand for $d=2$ bosonic $(\mu, \nu, \ldots)$ and $O(n)(\mathrm{a}, \mathrm{b}, \ldots)$ fermionic indices of the worldsheet; $\mathrm{P}^{\mathcal{M N}}=(-1)^{\mathcal{M N}+1} \mathrm{P}^{\mathcal{N} \mathcal{M}}$ is a Lagrange multiplier; $A_{\mathcal{M}}$ is a supersymmetric generalization of the "electromagnetic" field $A_{\mu}$ in (7);

$$
B_{\mathcal{M N}}=i \partial_{\mathcal{M}} X^{m} \partial_{\mathcal{N}} \bar{\Theta} \gamma_{m} \Theta+(-1)^{\mathcal{M N}+1} i \partial_{\mathcal{N}} X^{m} \partial_{\mathcal{M}} \bar{\Theta} \gamma_{m} \Theta
$$


defines the pull-back onto the super worldsheet of the Wess-Zumino two-form $B=d X^{m} \wedge$ $d \bar{\Theta} \gamma_{m} \Theta$, which is the same as that of the heterotic string [2], and which, in general, can be introduced in the case of a null superstring as well; and

$$
F_{\mathcal{M N}}=\left(V_{\mathcal{M}} \Omega_{\mathcal{N}}+(-1)^{\mathcal{M N}+1} V_{\mathcal{N}} \Omega_{\mathcal{M}}\right) D_{a} \bar{\Theta} \gamma_{m} D_{a} \Theta^{\mathcal{L}}\left(\partial_{\mathcal{L}} X^{m}-i \partial_{\mathcal{L}} \bar{\Theta} \gamma_{m} \Theta\right)
$$

is introduced in such a way that, with taking into account the twistor condition $D_{a} X^{m}-$ $i D_{a} \bar{\Theta} \gamma_{m} \Theta=0$, its corollaries and the constraint (18), $d B=d F$ on the mass shell [2].

In (35) superfields $V_{\mathcal{M}}, \Omega_{\mathcal{M}}$ and $\Omega^{\mathcal{M}}$ generalize the bosonic vectors (8). In particular,

$$
V_{\mu}\left(D_{a} V_{a}^{\mu}\right)=1, \quad \Omega_{\mu}\left(D_{a} V_{a}^{\mu}\right)=0, \quad V_{a}=-i V_{a}^{\mu} V_{\mu}, \quad \Omega_{a}=-i V_{a}^{\mu} \Omega_{\mu}
$$

As in the bosonic case above one may convince oneself that the induced worldsheet metric is not degenerate anymore, $V_{\mathcal{M}}$ and $\Omega_{\mathcal{M}}$ may be identified with the elements $E_{\mathcal{M}}^{+}$ and $E_{\mathcal{M}}^{-}$of the superzweibein matrix on the worldsheet, and the action (23) plus (33) turns out to be completely the same as the heterotic string action of Ref. [2]. The string tension $T$ arises as an integration constant of the equation of motion:

$$
\partial_{\mathcal{M}} \mathrm{P}^{\mathcal{M N}}=0
$$

from which, by gauge fixing the local transformations $\delta \mathrm{P}^{\mathcal{M N}}=\partial_{\mathcal{L}} \Xi^{\mathcal{L M N}}$ (where $\Xi^{\mathcal{L M N}}$ is a graded antisymmetric parameter), one may find that the only nontrivial component of $\mathrm{P}^{\mathcal{M N}}$ is $p^{\mu \nu}=\varepsilon^{\mu \nu} T \eta^{n}$ (see [2] for the details).

In conclusion we have constructed the doubly supersymmetric model for $N=1$, $D=3,4,6$ and 10 null superstrings which elucidates from somewhat different point of view the meaning of the both ingredients of the twistor-like heterotic string formulation [15], 2]: the twistor-like null superstring action corresponds to the "geometro-dynamical" part of the twistor-like heterotic string action, while introducing an "electromagnetic" superfield propagating in the null super worldsheet and taking into account the WessZumino term á la Townsend and Galperin et. al. leads to the string tension generation and results in the model which is proved to be classically equivalent to the heterotic string.

\section{Acknowledgments}

D.S. is grateful to the European Community for financial support under the contract CEE-SCI-CT92-0789, the University of Padova and INFN (Sezione di Padova), and in particular Prof. M. Tonin for kind hospitality in Padova.

I.B., D.S. and D.V. are grateful to A. Zheltukhin for stimulating discussion. 


\section{References}

[1] J.A.De Azcàrraga, J.M.Izquierdo and P.K.Townsend, Phys. Rev. D45 (1992) 3321;

P. K. Townsend, Phys. Lett. 277B (1992) 285;

E.Bergshoeff, L.A.J.London and P.K.Townsend, Class. Quantum Grav. 9 (1992) 2545 .

[2] F. Delduc, A. Galperin, P. Howe and E. Sokatchev, Phys. Rev. D47 (1992) 578.

[3] A. Shild, Phys. Rev. D16 (1977) 1722;

A. Karlhede and U. Lindström, Class. Quantum Grav. 3 (1986) L73;

A. P. Balachandran, F. Lizzi and G. Sparano, Nucl. Phys. B263 (1986) 608; Nucl. Phys. B277 (1986) 359.

[4] A. Zheltukhin, Sov. Phys. JETP Lett. 46 (1987) 208; Sov. J. Nucl. Phys. 48 (1988) 375.

[5] A. Zheltukhin, Theor. Math. Phys. 77 (1988) 1264; Sov. J. Nucl. Phys. 51 (1990) 950.

[6] F. Lizzi, B. Rai, G. Sparano and A. Srivastava, Phys. Lett. 182B (1986) 326; Phys. Rev. Lett. 182 (1987) 376;

J. Gamboa, C. Ramirez and M. Ruiz-Altaba, Phys. Lett. 225B (1989) 335.

[7] I. A. Bandos and A. A. Zheltukhin, Sov. Phys. JETP Lett. 51 (1990) 547; Sov. Phys. JETP Lett. 53 (1991) 5; Phys. Lett. 261B (1991) 245; Theor. Math. Phys. 88 (1991) 358; Fortsch. der Phys. (1993) in press.

[8] A. Barcelos-Neto and M. Ruiz-Altaba, Phys. Lett. 228B (1989) 193.

[9] U. Lindström, B. Sundborg and G. Theodoridis, Phys. Lett. 253B (1991) 319; Phys. Lett. 258B (1991) 331.

[10] U. Lindström and M. Roc̃ek, Phys. Lett. 271B (1991) 79.

[11] M. B. Green, J. H. Schwarz and E. Witten, Superstring Theory, CUP, 1987.

[12] D. P. Sorokin, V. I. Tkach and D. V. Volkov, Mod. Phys. Lett. A4 (1989) 901;

D. P. Sorokin, V. I. Tkach, D. V. Volkov and A. A. Zheltukhin, Phys. Lett. 216B (1989) 302;

D. P. Sorokin, Fortsch. der Phys. 38 (1990) 302;

A. I. Gumenchuk and D. P. Sorokin, Sov. J. Nucl. Phys. 51 (1990) 350; 
V. A. Soroka, D. P. Sorokin, V. I. Tkach and D. V. Volkov, Int. J. Mod. Phys. A7 (1992) 5977;

A. I. Pashnev and D. P. Sorokin Class. Quantum Grav. 10 (1993) 625.

[13] D. V. Volkov and A. A. Zheltukhin, Letters in Math. Phys. 17 (1989) 141; Nucl. Phys. B335 (1990) 723.

[14] N. Berkovits, Phys. Lett. 232B (1989)184; 241B (1990) 497; Nucl. Phys. B350 (1991) 193; B358 (1991) 169; B395 (1993) 77.

[15] M. Tonin, Phys. Lett. 266B (1991) 312; Int. J. Mod. Phys. A7 (1992) 6013;

S. Aoyama, P. Pasti and M. Tonin, Phys. Lett. 283B (1992) 213;

P. Pasti and M. Tonin, Preprint DFPD/93/TH/07, Padova, 1993.

[16] P. Howe and P. Townsend, Phys. Lett. 259B (1991) 285.

[17] F. Delduc and E.Sokatchev, Class. Quantum Grav. 9 (1991) 361;

A. Galperin and E. Sokatchev, Phys. Rev. D46 (1992) 714;

E. A. Ivanov and A. A. Kapustnikov, Phys. Lett. 267B (1991) 175;

F. Delduc, E. Ivanov and E. Sokatchev, Nucl. Phys. B384 (1992) 334;

A. S. Galperin, P. S. Howe and K. S. Stelle, Nucl. Phys. B368 (1992) 281;

F. Delduc, A. Galperin and E. Sokatchev, Ibid. B368(1992)143.

[18] A. Galperin and E. Sokatchev, Preprint JHU-TIPAC-93007, BONN-HE-93-05, 1993.

[19] J. P. Gauntlet Phys. Lett. 272B (1991) 25.

[20] V. Chikalov and A. Pashnev, Mod. Phys. Lett. A8 (1993) 285.

[21] S. J. Gates Jr. and H. Nishino, Class. Quantum Grav. 3(1986) 391;

J. Kowalski-Glikman, Phys. Lett. 180B (1986) 358;

R. Brooks, F. Muhammed and S. J. Gates Jr., Nucl. Phys. B268 (1986) 599; Class. Quantum Grav. 3 (1986) 745;

R. Brooks, Phys. Lett. 186B (1987) 313;

J. Kowalski-Glikman, J. W. van Holten, S. Aoyama and J. Lukierski, Phys. Lett. 201B (1987) 487;

A. Kavalov and R. L. Mkrtchyan, Preprint Yer.PhI 1068(31)-88, Yerevan, 1988 (unpublished).

[22] L. Brink, S. Deser, B. Zumino et. al. Phys. Lett. 64B (1976) 435;

L. Brink, P. Di Vecchia and P. Howe, Nucl. Phys. B118 (19977) 76. 